\title{
Trustworthiness
}

\section{A Developmental Perspective}

\begin{abstract}
Trust and trustworthiness are important pillars of interpersonal, societal, and economic functioning. We provide an overview of how trustworthiness develops across the lifespan.

Previous studies point to an increase in trustworthiness during childhood; relatively stable levels throughout adolescence and adulthood; and some evidence suggests a further increase in old age. Young children's lower levels of trustworthiness are mirrored by differences in fundamental motives that drive social behavior, such as concerns for reciprocity and fairness. While reciprocal tendencies emerge early, young children show less fairness concerns. This pattern can be linked to the development of certain cognitive abilities that are necessary to engage in social behaviors beyond indiscriminate selfishness. Children need to exert self-control to resist the temptation of keeping all resources to themselves and they need to engage in perspective-taking to appreciate the negative consequences of their selfishness. Thus, both social and cognitive development should be considered when studying age differences in trustworthiness.
\end{abstract}

\author{
Bastian Jaeger \\ Tilburg University \\ b.jaeger@uvt.nl \\ Anthony M. Evans \\ Tilburg University \\ Ilja van Beest \\ Tilburg University
}

JEL-Code: C720, C91, D01

Keywords: trustworthiness, age, reciprocity, fairness.

Manuscript version: 25/07/2016

This paper appears as an entry in The SAGE Encyclopedia of Lifespan Human Development.

Please cite as follows:

Jaeger, B., Evans, A. M., \& van Beest, I. (2018). Trustworthiness. In M. Bornstein (Ed.), The SAGE

Encyclopedia of Lifespan Human Development. (pp. 2271-2272). Thousand Oaks, CA: Sage. 


\section{Trustworthiness: A Developmental Perspective}

Social relationships depend on trustworthiness - we rely on colleagues to contribute to group projects; we hope that the products we buy work as advertised; and we expect friends to live up to their promises. In other words, social life means trusting others to live up to our expectations. In addition, we expect others to conform to countless (often implicit) rules shared within a culture or society. Trust between two parties can be mutually beneficial, and unsurprisingly, trustworthiness is an important factor in organizational success, economic growth, and the functioning of personal relationships. Parents may hope that their children will grow up to be deserving of trust, but can we expect children to be trustworthy? And does trustworthiness increase (or decrease) with age?

This entry provides an overview of age differences in trustworthiness and discusses the mechanisms underlying the emergence of trustworthy behavior. In short, trustworthiness is related to the development of social motives and cognitive skills. Social motives, such as reciprocity and fairness, are fundamental preferences regarding how resources are shared in groups. These motives depend on certain cognitive skills, such as the abilities to take other perspectives and resist temptations. This means that trustworthy behavior is determined by both upbringing and cognitive development.

\section{Defining and Measuring Trustworthiness}

Trust is the willingness to make oneself vulnerable to other people based on expectations of their future behavior. Trustworthiness, in turn, is the positive reciprocation of the trust extended from one person (the trustor) to another (the trustee). These definitions underline the deep connection between trust and trustworthiness — trust is based on expected trustworthiness, 
and the mere act of trusting can elicit trustworthiness through reciprocity. Without trustworthiness, there can be no benefit to trusting in others.

Trust and trustworthiness can be measured using the trust game, an economic interaction involving two parties, the trustor and the trustee. To begin, the trustor receives a valuable resource (adults receive money, children receive stickers or candy). The trustor decides to send some or all of this resource to the trustee. The amount sent is then tripled by the experimenter. In the second step, the trustee decides how to share this tripled resource with the trustor. The trustee can send back some of the tripled resource, or keep all of it. Reciprocated trust represents a gain for both players. However, it is risky for the trustor to send money, as the trustee faces the temptation to keep everything. While the first transfer is a measure of trust, the second transfer represents trustworthiness.

The trust game can be analyzed from a game-theoretic perspective: In game theory, social interactions are represented and analyzed according to the principles of rational choice. Basic game-theoretic models assume that people are strictly self-interested; in other words, they only care about maximizing personal outcomes. In this view, a selfish trustee always keeps everything. The trustor should anticipate this reaction and withhold trust. Thus, game theory predicts that trust and trustworthiness will not be observed when strangers interact. Yet, countless experiments have demonstrated that both behaviors are common in the trust game.

\section{Trustworthiness Across the Lifespan}

Researchers have used the trust game to ask how trustworthiness changes across the lifespan. One large-scale study looked at age groups that ranged from 8-year-olds to retired adults over 65. Trustworthy behavior was apparent in every age group, but the degree of trustworthiness (the amount returned in the trust game) steadily increased with age. Young 
children, 8- and 12-year-olds, were the least trustworthy; 16-year-olds and adults were moderately trustworthy; and retired adults were the most trustworthy. Other studies focusing on the differences between specific age groups confirm this general pattern. Trustworthy behavior increases during childhood, peaks in adolescence, and remains stable across adulthood. Older adults, those over 65 years of age, are sometimes found to be even more trustworthy, but evidence for this increase is mixed. Why do young children struggle to honor trust? Their behavior may be explained by the development of two social motives - concerns for reciprocity and fairness.

The trustor's decision to send resources directly benefits the trustee, creating an obligation for the trustee to "return the favor". This norm of reciprocity has been identified in every studied society and has been theorized to be one of the driving forces behind the evolution of cooperation. From an evolutionary perspective, humans may be particularly equipped to engage in reciprocal behavior. Indeed, even 4-year-olds have a basic understanding of reciprocity. Young children (5- to 7-year-olds) prefer to help those who have helped them in the past.

Given the early emergence of reciprocity, low levels of trustworthiness among children might seem surprising. However, trustworthiness is also motivated by fairness concerns. Irrespective of reciprocity, trustees can simply be motivated to establish a fair split of the available resources. Experiments in which children were able to keep or share a resource have demonstrated that sharing generally increases with age. While 3-to 4-year-olds are still overwhelmingly selfish, many 7-to 8-year-olds prefer to share. Younger children would rather accept an unfair split than have nothing at all. Older children are more willing to punish their 
partners for their unfair behavior. Thus, young children attach less importance to fairness and focus more on maximizing their own outcomes.

Together, these results suggest that there are different developmental trajectories for the social motives underlying trustworthiness. Reciprocal behavior emerges early, but concerns over fairness develop with age. Young children are more selfish when sharing is costly for them, which can account for the increase in trustworthiness documented across childhood.

\section{Trustworthiness and Cognitive Abilities}

Reciprocity and fairness are powerful motives. As children mature, they learn to act according to these rules through parenting and direct social encounters. For example, a child's relationship with her parents shapes how she behaves in social interactions in later life. Children turn to their caregivers for help in times of distress, and children who know that they can depend on their parents develop a secure attachment to them. These feelings of security and positive expectations influence expectations about other people in general. Accordingly, securely attached individuals are more trusting and also believe others to be more trustworthy. Children may also learn how to navigate social situations through interactions with their brothers and sisters. Siblings often compete for limited resources like their parents' attention, and the need to resolve these conflicts drives the emergence of social motives. Finally, reciprocity and fairness depend on the development of cognitive skills. These skills provide children with the tools needed to engage in a variety of social behaviors that require more complex processes than indiscriminate selfishness.

One example of a necessary cognitive development is the emergence of self-control, the capacity to inhibit or suppress an impulse. Self-control shows dramatic improvement in the second year of life. For children, the temptation to keep all toys, stickers, or candy to themselves 
must loom large. To act in a fair and trustworthy manner, children must be able to override their selfish impulse first. Correspondingly, preschoolers high in self-control were judged to be more trustworthy by both their classmates and teachers.

Another example is the emergence of theory of mind, the ability to recognize other people as separate entities with their own mental states, such as emotions, motives, and thoughts. This ability develops between the ages of 3 and 9. Children must engage in perspective-taking to fully appreciate the negative impact of selfish behavior. If these faculties are not yet present, untrustworthy behavior might not reflect intentional selfishness but simply the inability to consider the social consequences of selfish behavior. Accordingly, individual differences in empathy among 3-to 5-year olds have been shown to predict sharing behavior.

The prevalence of trustworthiness varies considerably across the lifespan. The general increase in trustworthiness with age can be explained by the emergence of different social motives underlying trustworthy behavior (e.g., concerns for reciprocity and fairness). Trustworthiness also requires certain cognitive skills, such as the abilities to engage in selfcontrol and perspective-taking. Thus, understanding when and to what extent a person will be trustworthy depends on socialization and on cognitive development. 


\section{Further Readings}

Evans, A. M., Athenstaedt, U., \& Krueger, J. I. (2013). The development of trust and altruism during childhood. Journal of Economic Psychology, 36, 82-95.

Sutter, M., \& Kocher, M. G. (2007). Trust and trustworthiness across different age groups. Games and Economic Behavior, 59(2), 364-382.

Van Lange, P. A. M., Otten, W., De Bruin, E. M., \& Joireman, J. A. (1997). Development of prosocial, individualistic, and competitive orientations: theory and preliminary evidence. Journal of Personality and Social Psychology, 73(4), 733-746. 